\title{
Study on Capturing Functional Requirements of the New Product Based on Evolution
}

\author{
Fang Liu ${ }^{1}$, Liya Song ${ }^{2}$, Zhonghang Bai ${ }^{1}$, and Peng Zhang ${ }^{1}$ \\ ${ }^{1}$ School of Mechanical Engineering, Hebei University of Technology, \\ Tianjin, PRC \\ ${ }^{2}$ Experiment Center of Electron and Communication, shijiazhuang university of \\ ecomomics, Shijiazhuang, PRC \\ \{Fang. Liu, Liya.Song, Zhonghang.Ba, Peng. Zhang, liufangzzh\}@sina.com
}

\begin{abstract}
In order to exist in an increasingly competitive global marketplace, it is important for corporations to forecast the evolutionary direction of new products rapidly and effectively. Most products in the world are developed based on the design of existing products. In the product design, capturing functional requirements is a key step. Function is continuously evolving, which is driven by the evolution of needs and technologies. So the functional requirements of new product can be forecasted based on the functions of existing product. Eight laws of function evolution are put forward in this paper. The process model of capturing the functional requirements of new product based on function evolution is proposed. An example illustrates the design process.
\end{abstract}

Keywords: Evolution, functional requirement, law, TRIZ.

\section{Introduction}

In axiomatic design, the product design process is made up of four domains: the customer domain, the functional domain, the physical domain, and the process domain [1]. Each domain has respectively design tasks, which are customer requirement, functional requirement, design parameter and process variable. The product design is the process of zigzagging mapping between the four domains. In the functional domain, the customer needs are specified in terms of functional requirements, which are implemented by product structure. So customers and designers are connected by functional requirements. Though the representations of customer requirements are different, their aims are to achieve corresponding functions by products. The function is the direct cause of product existence.

At present time, the functional requirements are directly produced by analyzing customer needs in the product design, and customer needs are commonly obtained by questionnaires, interview or comparing correlative products. These methods have some limitations. The customer requirements are usually expressed by natural language, which are very vague. So the efficiency of capturing functional requirements is poor. Product is continuously evolving [2]. In order to exist in an increasingly competitive global marketplace, it is important for corporations to forecast the 
evolutionary direction of new products rapidly and effectively. Most products in the world are developed based on the design of existing products. So new product can be forecasted by the evolutionary analyzing of existing product.

In this paper, the function evolution is discussed, and eight laws of function evolution are put forward. The goal of this paper is to capture the functional requirements of new product by analyzing the function evolution of existing product. A process model is proposed, which is illustrated using an industrial example.

\section{The Laws of Function Evolution}

TRIZ is Russian acronym for The Theory of Inventive Problem Solving [3]. It is put forward by G.S.Altshuller and other researchers of former Russia based on the analysis of 2,500,000 world-wide patents. TRIZ specially deals with innovation and conceptual design [3]. It has already formed a serial of tools used for resolving problems, such as technology forecasting.

In TRIZ the technological systems evolve toward ideality according to forecasted evolution patterns. The evolution of technological systems has three levels: the evolution of needs, the evolution of functions and the evolution of technologies. Petrov proposed five laws of needs evolution, which were shown as follows [4]:

Law 1: idealization of needs;

Law 2: dynamization of needs;

Law 3: coordination of needs;

Law 4: integration of needs;

Law 5: specialization of needs.

The TRIZ technology evolution has different versions [5-7]. Fry and Rivin concluded nine laws of technology evolution based on the former TRIZ research, which were shown as follows:

Law 1: increasing degree of ideality;

Law 2: non-uniform evolution of subsystems;

Law 3: transition to a higher-level system;

Law 4: increasing dynamism;

Law 5: transition to micro-level;

Law 6: completeness;

Law 7: shortening of energy flow path;

Law 8: increasing substance-field interactions;

Law 9: harmonization of rhythms.

Every law includes several lines of technology evolution, and every line includes several states. Lines of technology evolution provide detailed descriptions of how a system evolves and therefore provide even greater predicting power.

Petrov proposed four laws of function evolution, which were idealization of functions, increasing the degree of function dynamics, transition to mono- or polyfunctionality and coordination of functions. The laws of function evolution are similar to the laws of demand evolution, but they are regarded with respect to functionality. Product is the implementation of required functions. The embodiment of function is 
how customer needs are satisfied by product. Moreover the function is implemented by special technology or structure. So the function is evolving with the evolution of need and technology. In this paper eight laws of function are concluded based on the evolution of need and technology, which are idealization of functions, integration of functions, specialization of functions, completeness of functions, dynamization of functions, controllability of functions, non-uniform evolution of functions and coordination of functions. Idealization of functions is performed through integration of functions, specialization of functions, completeness of functions, dynamization of functions, controllability of functions, non-uniform evolution of functions, and the latter six laws are achieved by coordination of functions as shown in Fig.1.

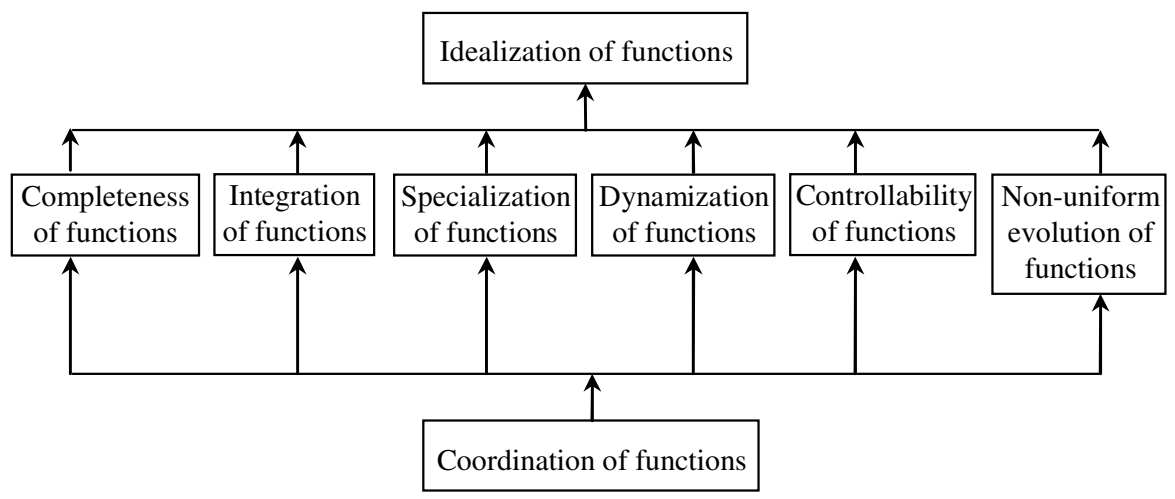

Fig. 1. The laws of function evolution

\section{Idealization of functions}

Idealization of functions presupposes the improvement of the functions' quantity, functions' quality, and the reduction of harmful actions. Degree of idealization of functions could be presented in the following form:

$$
I=\frac{\sum_{i=1}^{n} U F_{i}}{\sum_{j=1}^{m} H F_{j}} \Rightarrow \infty
$$

Where:

$$
\begin{aligned}
& I-\text { degree of idealization of function; } \\
& U F-\text { the useful function; } \\
& H F-\text { the harmful function; } \\
& i \text { - the quantity of useful functions; } \\
& j \quad \text { - the quantity of harmful functions. }
\end{aligned}
$$

The degree of idealization tending to infinity is the trend of idealization of functions. Four methods could accomplish the idealization of functions: increasing the quantity of useful functions, improving the quality of useful functions, decreasing the quantity of harmful functions and reducing the degree of side effect. 


\section{Completeness of functions}

Completeness of functions involves the completeness of the basic function and the technological system's functions.

In TRIZ a basic function comprises three elements: two substances and one field. Function can not be implemented if any one is absent, i.e. the function is not complete.

A technological system consists of four principal parts: working means, transmission, engine, and control means. A working means is a component that directly performs the primary function of the system. A transmission transforms the energy produced by the engine into the energy that can control the working means. A control means allows for changing the parameters of the other principal parts.

3. Integration of functions

The integration is performed in such a way that the useful functions are summed up or intensified, while the harmful functions are either mutually compensated or minified. There are several ways of integration:

- Integration of similar or identical functions;

- Integration of opposite functions;

- Integration of heterogeneous functions;

- Generation of similar functions with shifted characteristics;

- Generation of competing or alternative needs.

4. Specialization of functions

Specialization of function is to make the universal function specified and achieve it more accurately and at a higher quality level. The specialization of functions is performed in the following sequence:

- Select the most important function;

- Develop this function;

- Provide for better conditions of satisfying this function.

- Dynamization of functions

Dynamization of functions indicates the flexibility of the function, which can presupposes the following trend:

- The functions get adapted for the changes in time, in space, in structure, according to a definite condition.

- The functions get adapted for definite location, a group of people or a particular person.

- Eliminate human involvement in labor processes.

5. Controllability of functions

Controllability of functions is to intensify the interactions between the controlling functions and other functions, which results in the increasing substance-field interactions. Controllability of functions is achieved in several ways:

- Increase the number of elements in complex substance-field.

- Replace an uncontrollable or poorly controllable field with a better controllable field.

- Increase degree of flexibility of the substance-field elements. 


\section{Non-uniform evolution of functions}

In the technological system, there are complicated interactions between functions. Each function is in the respective evolutionary process. The development of the functions of the technological system is non-uniform. The more complicated the technological system is, the more high the non-uniform degree is. The non-uniform evolution of functions leads to the contradictions in the technological system. Eliminating contradictions may make the system harmonious.

\section{Coordination of functions}

Coordination of functions involves the coordination between subsystems in parameters, structure, conditions, space, and time. Coordination could be dynamic. Coordination of functions can be understood that there are dis-coordinations in the system, which should be eliminated.

\section{The Process Model of Capturing Functional Requirement Based on Function Evolution}

Function is continuously evolving, which is driven by the evolution of needs and technologies. So the functional requirements of new product can be forecasted by using the laws of function evolution based on the functions of existing product. The process model of capturing the functional requirements of new product based on function evolution is shown in Fig.2, which includes the following five steps.

Step 1: select an existing product as the prototype of future development.

Step 2: analyze the existing function requirements and construct function tree.

Functional requirement analyzing is rooted in the theory of value engineering. It hierarchically decomposes the overall product function into sub-functions according to logical relationships on the basis of function definition. Function is a statement to describe the transformation between inputs and outputs, aiming to achieve the designer's purpose [8]. The logical relationships among functions can be shown by constructing function tree.

Step 3: identify the disadvantages or problems in current system.

In this step, the relationships between functions and components are analyzed and function model is constructed according TRIZ substance-field. Analyze the inadequate actions and the harmful actions existed in current system, and then identify the disadvantages or problems.

Step 4: identify the disadvantages or problems in future system and the evolutionary resources.

In this step, the current system is analyzed by using laws of function evolution one by one, and then the disadvantages or problems in future system are identified.

The evolution of system is a result of the accumulation of knowledge, contribution to the increasingly efficient utilization of resources, both internal and external [9]. A system's evolution slows down and can eventually stop due to the exhaustion of the available resources. Evolutionary resources consist of the knowledge, abilities, skills, etc. obtained as a result of prior evolution and capable of enabling a subsequent evolutionary step [9]. The evolutionary resources can be divided into four categories as follows [9]: 
- Resources developed in the given area starting at the moment the system was created.

- Resources from other areas of technology that can be utilized in the system.

- Resources from the areas of sociology, marketing, and psychology.

- Knowledge about evolution.

The identified disadvantages or problems in future system and evolutionary resources show the possible directions of new product development.

Step 5: classify and systemize the current and future disadvantages or problems, and then identify the latent functional requirements of the new product.

Select an existing product

- Analyze the existing functional requirements

- Construct function tree

- Analyze the relationships between functions and components

- Establish the system function model

- Identify the disadvantages or problems in the current system

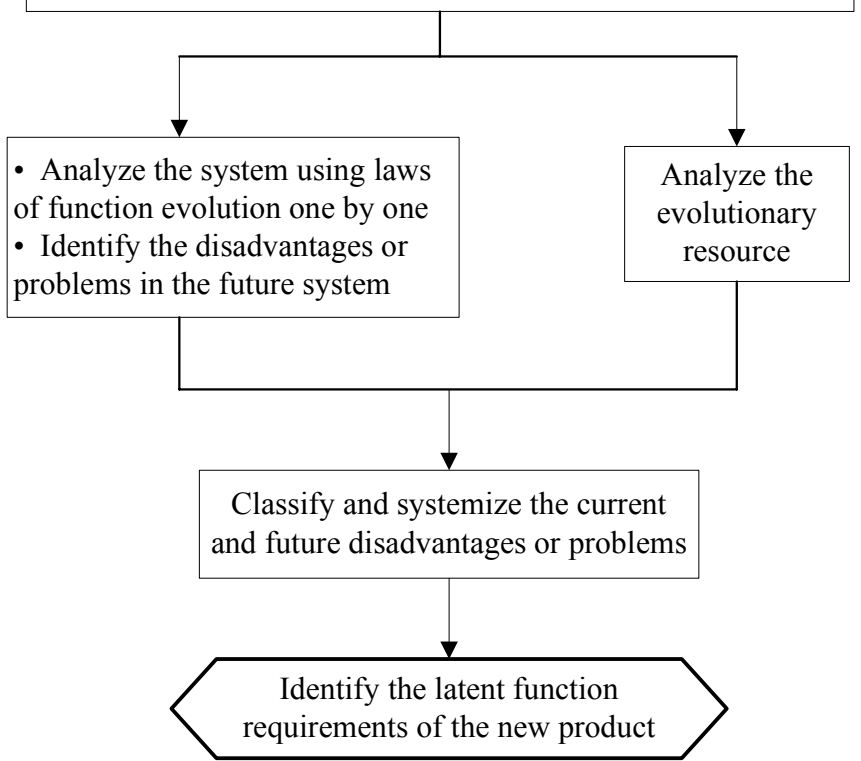

Fig. 2. The process model of capturing functional requirements based on function evolution 


\section{Case Study}

Heat meter is an instrument that measures the quantity of heat absorbed or released by a loop of heat flow. It is widely accepted by customers as an evidence of heating cost. Heat meter is composed of flowmeter, calculator and two temperature sensors. When water flows into the system, flowmeter measures the flux and two temperature sensors detect the water temperatures of inlet and outlet respectively. Calculator receives the information of flux and two temperatures and calculates the quantity of heat that exhausted by the heating system.

According to the type of flowmeter, the heat meter can be divided into three kinds: Mechanical, electromagnetic and ultrasonic. Due to the advantage of the mechanical heat meter, i.e. low prices, high-precision measurement and so on, the mechanical heat meter is widely used. For the mechanical heat meter, the flux is calculated by measuring the mechanical rotation of impeller. Mechanical heat meter can be also divided into two kinds: single channel and multi-channel. At present, mechanical heat meter with multi-channel is widely used due to its stable performance. The sketch of mechanical heat meter is shown in fig. 3 .

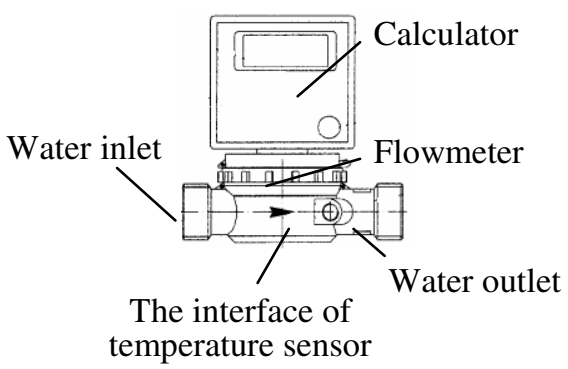

Fig. 3. The sketch of mechanical heat meter

Construct function tree by analyzing the existing functional requirements of heat meter as shown in fig.4. Analyze the relationships between functions and components, and then construct the function model as shown in Fig.5.

It can be concluded by analyzing the function model that there are some disadvantages in the existing heat meter. Though a filter is generally installed in the water inlet, heat meter is often blocked by the impurities in the water, which have effect on the precision measurement and cause non-normal working of the heat meter. Moreover, the calculator consumes the electrical energy, which is supplied by batteries.

Analyze the evolutionary directions of heat meter by using the laws of functions. According to the dynamization of function, the new product could filter the impurities automatically. According to the integration of function, the new product could not only measure the quantity of heat but also clean the impurities. According to idealization of functions, the working of a new product does not need extra energy supplying, and it can adapt the water with poor quality. Next step, the available evolutionary resources are analyzed. The effect of centrifugal may be used to implement the function of clean the impurities automatically. Moreover, through development a new component, the electric energy may be generated by using the high-speed rotation of the impeller. 


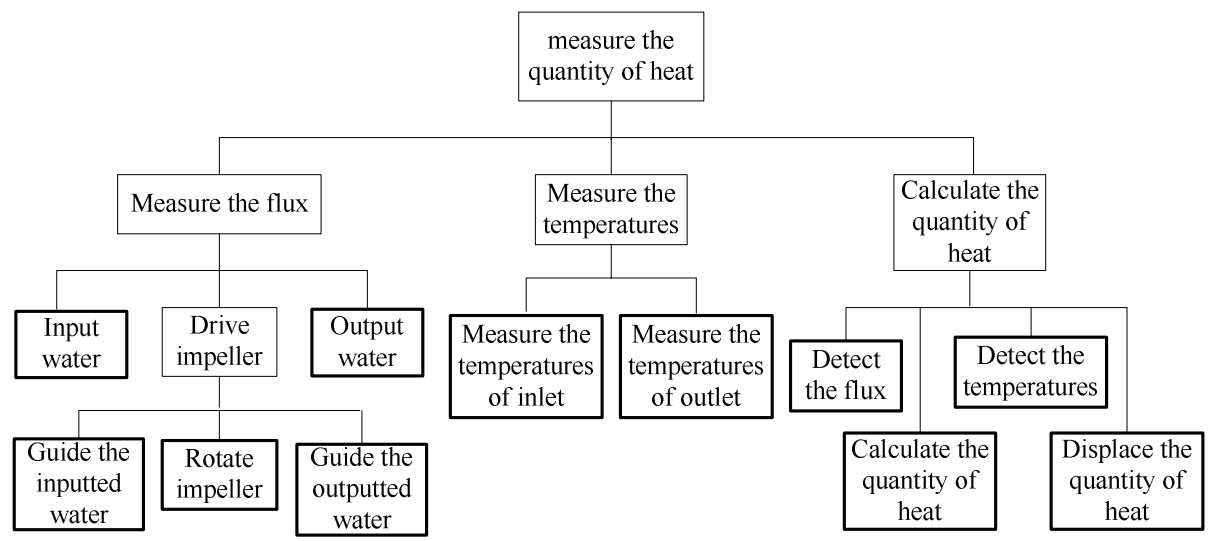

Fig. 4. Function tree
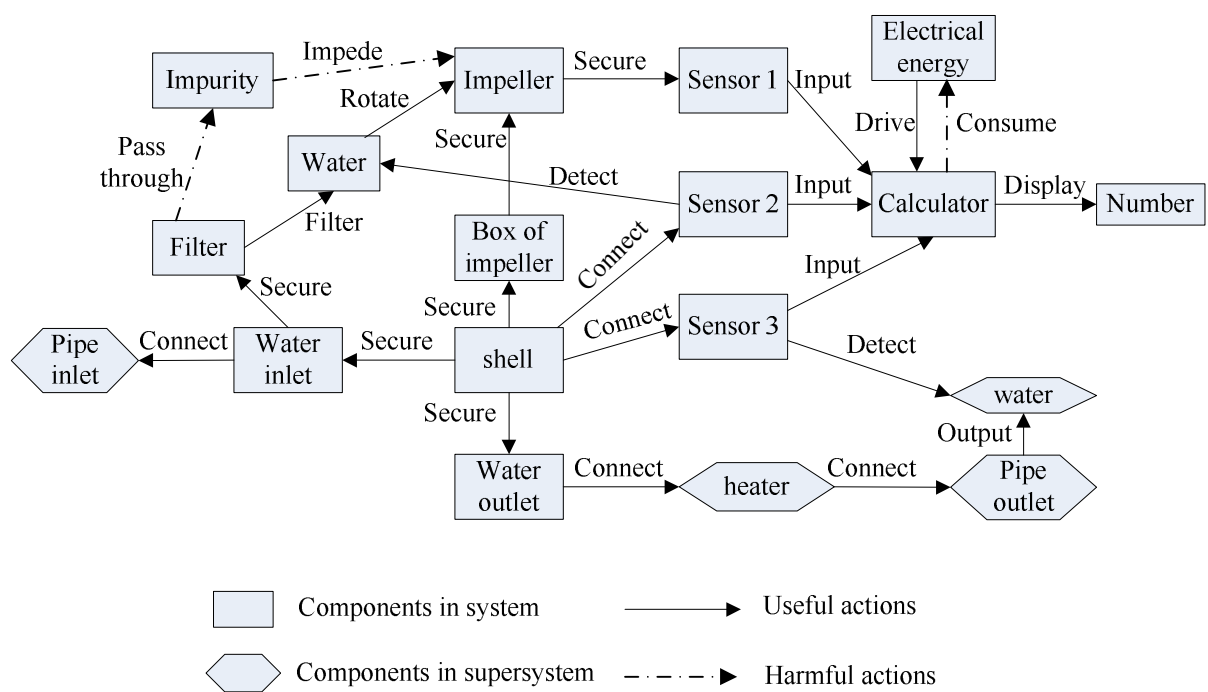

Fig. 5. Function model

The functional requirements can be identified by systemizing the above analysis. The basic functional requirements are measuring flux, measuring temperature and calculating quantity of heat. The latent functional requirements are clean the impurities automatically and generating electric energy by itself.

\section{Conclusion}

Most products in the world are developed based on the design of existing products. New products can be forecasted by evolutionary analyzing of existing products. On the basis of the evolution of need and technology, this paper discusses the evolution 
of function. Eight laws of function evolution are proposed. A process model of capturing functional requirements of new product is put forward. The latent functional requirements of heat meter are identified, which illustrates the design model.

\section{Acknowledgment}

This research is supported in part by the Natural Science Foundation of Tianjin under Grant Numbers 07JCZDJC08900, the National Science and Technology Basic Project under Grant Numbers 2008IM030100, and the Natural Science Foundation of Hebei under Grant Number E2008000086. Any opinions or findings of this work are the responsibility of the authors, and do not necessarily reflect the views of the sponsors or collaborators.

\section{References}

1. Nam, P.S.: Axiomatic Design: Advances and Applications. Oxford University Press, New York (2001)

2. Tan, R.: Innovative design-TRIZ: theory of inventive problem solving. China machine press, Beijing (2002)

3. Altshuller, G.: The Innovation Algorithm, TRIZ, systematic innovation and technical creativity. Technical Innovation center, Worcester (1999)

4. Petrov, V.: Laws of development of needs (2006), http: / /www.triz-journal . com

5. Savransky, S.D.: Engineering of Creativity. CRC Press, New York (2000)

6. Fey, V.R., Rivin, E.I.: Guided technology evolution (TRIZ technology forecasting), http: //www.trizgroup.com/article2.html

7. Zusman, A., Zlotin, B., Zainiev, G.: An application of directed evolution, http://www.ideationtriz.com/Endoscopic_case_case_study.asp

8. Cao, G., Tan, R., Lian, B.: Research and implementation of product functional design based on effect. In: Second IFIP Working Conference on Computer Aided Innovation, Michigan, USA, pp. 67-76 (2007)

9. Boris, Z., Alla, Z.: The concept of resources in TRIZ: past, present and future,

http: / /www. ideationtriz.com/new/materials /

finalconceptresources.pdf 